\title{
Editorial: Meeting future energy demands with nanotechnology
}

Nitin Chopra PhD

Assistant Professor, University of Alabama, Tuscaloosa, USA

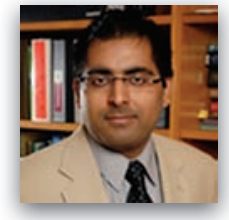

Developing alternative and renewable energy sources to replace or mitigate utilization of current energy sources, especially fossil or mineral fuel, is becoming critical (Dresselhaus and Thomas, 2001; Michaelides, 2012). In addition, research in the area of alternative and clean energy-generation methods will allow for safeguarding our environment from greenhouse gases, ozone layer depletion, and pollution. However, the high cost of manufacturing associated with devices and efficient material architectures are posing the biggest challenges for overcoming goals of alternative energy production. For example, recent statistics show that only $7 \%$ of the US energy market is composed of renewable energy sources, $8 \%$ nuclear, and $85 \%$ from fossil fuels (Serrano et al., 2009). Similar statistics are observed for other developed nations.

With progress in current technology, we have already marked our entry into a new era of "nano," which has been proving pivotal in positively impacting in the area of alternative energy and devices (Timp, 1998). Nanotechnology offers tools and approaches to develop new kinds of materials with unique properties that are critical for shaping the future energy market. Thus, in this issue of Nanomaterials and Energy, the focus is on material design for alternative energy systems such as photocatalysis and charge storage platforms. Furthermore, current state-of-the-art and future challenges involved with the design of nanocatalysts and nanoscale supercapacitors are comprehensively reviewed.

Growth understanding of nanomaterials plays the most important role in developing alternative energy devices. There are several reasons for this:

(1) If the composition, phase, and structure of nanomaterials are controlled at growth stage, then it will be possible to achieve desired properties, surface chemistry, and size scale of ultimate energy devices based on such nanomaterials.

(2) If it is possible to control the growth in a way that novel material architectures are grown in-situ, then it is feasible to eliminate need for device integration and processing steps at the later stages of technology development.

(3) If the material chemistry is well-understood, then it serves twofold purposes; control of material architecture at atomic scale and manipulation of its charge transport properties to result in efficient energy generation, conversion, and storage routes.

The above three aspects have been meticulously demonstrated by three articles in this issue that are presented by Kalkan et al., Qian et al., and Ramadurai et al. It is quite interesting to note that as the nanoscale-sized architectures are needed to be controlled, we are also pushing the limits of lithography methods. This necessitates self-assembly approaches, where pattering of one kind of nanostructures takes place on the other kind. For example, the article by Kalkan shows the fabrication of nanowire-nanoparticle heterostructures, where a direct nucleation of gold nanoparticles on oxide nanowire substrate resulted in enhanced plasmonic and photocatalytic effects due to heterostructuring. This area of research has been emerging in last few years, where researchers have been exploring opportunities for nanowire-nanoparticle heterostructures in energy sectors (Li et al., 2011; Sharma et al., 2006; Shi and Chopra, 2011; Tak et al., 2009). However, Qian et al. and Ramadurai et al. present approaches to fabricate carbon nanotubes (CNT) architectures using two approaches (chemical vapor deposition [CVD] and fluidized bed methods). Both of these approaches have strong potential for allowing CNTs and CNTbased energy devices to enter into commercial markets. Control of CNT growth in CVD method has led to synthesis of CNT architectures (Meyyappan, 2005). The article by Qian et al. demonstrates nitrogen-doped Y-junction CNT architectures in a CVD growth method. The potential of CNTs with dopants as shown by Qian et al. is promising for their applications in batteries and supercapacitors.

This issue also includes two comprehensive review articles in the area of nanocatalysis and supercapacitors. The article by Veser et al. reviews developments in nanoscale catalysts for watergas shift reaction, which is critical to fuel processing. This article discusses nanocatalyst design strategies, catalytic reaction mechanisms, and challenges involved in this field. The article by Bakker et al. presents a detailed review of supercapacitors, pseudocapacitors, and batteries based on nanomaterials such as carbon or metal nanostructures, silicon nanomaterials, and ionic liquids.

With this third issue of Nanomaterials and Energy, I also invite our valuable readers to submit their original research work, 
review articles, and commentaries to the Journal. Nanomaterials and Energy aims to provide a unique platform to the scientific community working in energy storage, conversion, and harvesting to highlight their ground-breaking research.

\section{REFERENCES}

Dresselhaus MS and Thomas IL (2001) Alternative energy technologies. Nature 414: 332-337.

Li HY, Eastman M, Schaller R, Hudson W and Jiao J (2011)

Hydrothermal synthesis of CdS nanoparticle-decorated $\mathrm{TiO}(2)$ nanobelts for solar cell. Journal of Nanoscience and Nanotechnology 11: 8517-8521.

Meyyappan M (2005) Carbon Nanotubes: Science and Applications. CRC Press, New York, NY.

Michaelides EE (2012) Alternative Energy Sources. Springer, London, UK.
Serrano E, Rus G and Garcia-Martınez J (2009) Nanotechnology for sustainable energy. Renewable and Sustainable Energy Reviews 13: 2373-2384.

Sharma J, Vivek JP, Vijayamohanan KP, Singh P and Dharmadhikari CV (2006) Directed organization of gold nanoclusters on silver nanowires: A step forward in heterostructure assembly. Applied Physics Letters 88: 193103.

Shi W and Chopra N (2011) Surfactant-free synthesis of novel copper oxide $(\mathrm{CuO})$ nanowire-cobalt oxide $(\mathrm{Co} 3 \mathrm{O} 4)$ nanoparticle heterostructures and their morphological control. Journal of Nanoparticle Research 13: 851-868.

Tak Y, Hong SJ, Lee JS and Yong K (2009) Solution-based synthesis of a CdS nanoparticle/ZnO nanowire heterostructure array. Crystal Growth and Design 9: 2627-2632.

Timp G (1998) Nanotechnology. Springer, New York, NY. 